\title{
Complete laparoscopic radical resection of hilar cholangiocarcinoma: technical aspects and long-term results from a single center
}

\author{
Jingdong Li ${ }^{1,2}$, Yongfu Xiong ${ }^{1,2}$, Gang Yang ${ }^{1}$, Lixing Zhang ${ }^{1}$, Muhammad Riaz ${ }^{1}$, Jian X $\mathrm{X}^{1}$, Qiang Li ${ }^{1}$, Zhaohui Tang ${ }^{3}$ \\ ${ }^{1}$ Department of Hepatobiliary Surgery, Affiliated Hospital of North Sichuan Medical College, Nanchong, China \\ ${ }^{2}$ Institute of Hepato-Biliary-Pancreatic-Intestinal Disease, North Sichuan Medical College, Nanchong, China \\ ${ }^{3}$ Department of General Surgery, Xinhua Hospital, Shanghai Jiao Tong University School of Medicine, Shanghai, China
}

Videosurgery Miniinv

DOI: https://doi.org/10.5114/wiitm.2020.97363

\begin{abstract}
Introduction: The landscape of surgical treatments for hepatobiliary disease was significantly changed after the advent of laparoscopy. Many kinds of complex laparoscopic procedures can be routinely performed at present, but radical resection of hilar cholangiocarcinoma (HC) by laparoscopy is still highly contentious.

Aim: To describe our primary experience with laparoscopic radical resection for HC and determine the safety and feasibility of this procedure.

Material and methods: Between December 2015 and November 2019, 32 patients planned to undergo curative-intent laparoscopic resection of HC in our department. The perioperative and long-term outcomes of these patients were retrospectively analyzed.

Results: Laparoscopic surgery with radical resection was ultimately performed in 24 (75.0\%) patients; 3 (9.3\%) patients were found to be unresectable at the preliminary exploration stage, and $5(15.7 \%)$ patients converted from laparoscopy to laparotomy. The operation time and blood loss were $476.95 \pm 133.89 \mathrm{~min}$ and $568.75 \pm 324.01 \mathrm{ml}$, respectively. A negative margin was achieved in 19 (79.1\%) of the laparoscopy patients. Three (12.5\%) patients were identified with microscopic positive margins, and 2 (8.4\%) patients underwent macroscopic residual tumor resection (R2). The length of postoperative stay was $23.3 \pm 11.7$ days. Severe morbidity occurred in 4 (16.6\%) patients. The actuarial 3-year overall survival and disease-free survival for patients who underwent laparoscopic surgery were $49.1 \%$ and $47.0 \%$, respectively.

Conclusions: Laparoscopic radical resection for HC is safe and feasible in experienced hands for highly selected patients but is still in its initial stages. When adequate oncologic resection is performed, the laparoscopic approach does not adversely influence the prognosis of the patient.
\end{abstract}

Key words: hilar cholangiocarcinoma, laparoscopy, radical resection.

\section{Introduction}

Hilar cholangiocarcinoma $(\mathrm{HC})$ is a rare but aggressive and complex pathological condition that is characterized by its location and spread to local and dis- tant organs [1-3]. With a prevalence of approximately 7000 cases per year in North America [2], less than $50 \%$ of these malignancies are resectable, and most of them succumb to the disease within a year of diag-

Address for correspondence

Jingdong Li MD, PhD, Department of Hepatobiliary Surgery, Affiliated Hospital of North Sichuan Medical College, Nanchong 637000,

China, e-mail: Li-Jingdong@hotmail.com; Zhaohui Tang MD, PhD, Department of General Surgery, Xinhua Hospital, Shanghai Jiao Tong

University School of Medicine, Shanghai 200000, China, e-mail: tangzhaohui@xinhuamed.com.cn 
nosis [4]. The incidence of $\mathrm{HC}$ has remained relatively stable over the past decades in Western countries but has rapidly increased in China over the same period [5]. In China, it is estimated that more than 65 thousand patients with $\mathrm{HC}$ are diagnosed annually $[6,7]$.

Radical resection has been well recognized as the most effective treatment for $\mathrm{HC}$ and is also the only choice that confers long-term survival [8-10]. However, curative surgical resection with negative margins can pose great challenges due to the involvement of the biliary confluence, including the right and left hepatic ducts, which are in close proximity to the portal vein, hepatic artery, and liver parenchyma [11]. It is clearly evident from the literature that aggressive curative surgical resection can offer a better long-term survival rate. For example, major liver resection combined with an extended portion of the hepatic duct could significantly improve the Ro resection rate $[12,13]$. Additionally, total removal of the caudate lobe has also been shown to decrease the recurrence rate and improve long-term survival $[14,15]$. Thus, in recent years, the operative treatment of $\mathrm{HC}$ has continued to develop, with the surgical strategy shifting from local excision of the affected bile duct to extended resections involving combined major liver resection. This also makes the surgical procedure of radical resection for $\mathrm{HC}$ technically more challenging and highly demanding. Thus, even conventional open surgical approaches can only be performed in selected centers with special expertise in the management of $\mathrm{HC}$.

The landscape of surgical treatments for hepatobiliary disease has significantly changed following the advent and evolution of laparoscopy [16]. In recent decades, we have witnessed the development of surgical devices, accumulated experience with laparoscopic surgery and spread of this minimally invasive surgery worldwide. Oncological outcomes for complicated laparoscopic maneuvers have been addressed in high-quality prospective studies for liver cancer [17, $18]$ and colon and rectal cancer [19, 20]. For example, laparoscopic liver resections are confirmed to be safe, with acceptable morbidity and mortality in experienced hands [21]. Additionally, the survival rates reported are comparable to those of open resection [21].

However, for the treatment of cancers occurring in the biliary tract, including $\mathrm{HC}$, a primary laparoscopic approach is still rejected by the surgical community $[22,23]$. In the past, the reasons cited have included oncological concerns, such as intraopera- tive peritoneal dissemination and possible port-site metastasis (PSM). Historically, there have indeed been an alarming number of reports of PSM at the initial utilization of laparoscopic surgery in biliary tract carcinoma. As shown in previous studies [24, 25 ], approximately $0.2-3.3 \%$ of patients were found to be gallbladder cancer positive postoperatively after laparoscopic cholecystectomy. Moreover, 50\% of gallbladder cancers are diagnosed incidentally [26]. Although their number is not large, the risk of PSM in these patients is estimated to be up to $14-30 \%$ [27]. There is obvious statistical bias that has resulted in a strong concern about the 'aerosol' effect of pneumoperitoneum. Increasing evidence suggests that the incidence of PSM is approximately $1.1 \%$ in laparoscopic cancer surgery, which is comparable to open surgery [28]. Nevertheless, the current recommendations for radical resection of biliary tract carcinoma, especially HC, stipulate that an open approach should be based largely on expert opinion rather than on evidence of superior outcomes.

Today, real concerns are associated with the technical demands involved in performing total laparoscopic radical resection. Technically, for radical resection of $\mathrm{HC}$, a surgeon must have experience in laparoscopic major liver resection with caudate lobectomy, hepatoduodenal lymphadenectomy and anastomosis between the hepatic duct and jejunum at the same time. Even for the experienced hepatobiliary surgeon, each of the abovementioned operative procedures is very challenging. Concerns about the safety, feasibility and outcomes have delayed the use of laparoscopic surgery in HC. In the past decade, with the accumulation of surgical experience in complicated hepatobiliary operations, the individual procedure that is essential for laparoscopic radical resection of $\mathrm{HC}$ has already been reported [21, 29]. Some studies recently described the application of minimally invasive surgery in patients with $\mathrm{HC}$ [30-36]. However, most of these studies have either enrolled a small number of patients or only included patients with $\mathrm{HC}$ in early stages. More importantly, few of the previous studies reported the long-term outcomes of $\mathrm{HC}$ patients following total laparoscopic radical resection.

\section{Aim}

The purpose of the present study is to determine the safety and feasibility of laparoscopic techniques in the treatment of $\mathrm{HC}$ patients and to investigate 
the oncological outcomes of patients who underwent total laparoscopic radical resection for $\mathrm{HC}$ at a single center specializing in hepatobiliary surgery at the current stage.

\section{Material and methods}

\section{Patient cohort and data collection}

Thirty-two patients who planned to undergo $\mathrm{Cu}$ rative-intent laparoscopic resection for $\mathrm{HC}$ between December 2015 and November 2019 were enrolled. No patient had any signs of metastasis on clinical assessment at the time of enrollment that were deemed unresectable. The patients were nonconsecutive. The inclusion criteria for laparoscopic curative-intent resection were basically the same as those for open surgery described in a previous study [37, 38]. Briefly, the criteria for nonresectability were defined as overt distant metastasis, intrahepatic metastasis, peritoneal seeding or para-aortic lymph node metastasis. Patients demonstrating the involvement of vascular structures in the hilum were not considered to be unresectable. Informed acknowledgement concerning the advanced nature of this procedure was obtained from all patients. All cases were reviewed and approved by the Medical Ethics Committee of the Affiliated Hospital of North Sichuan Medical College (NSMC). All operations were performed by one surgeon and his team. The protocol of this study was designed and conducted by the authors of this manuscript and was approved by the Institutional Review Board of the Affiliated Hospital of NSMC (No. NSMC2015-07). All the states that the research work has been reported in are in line with the PROCESS criteria.

\section{Preoperative management}

Multiple imaging assessments are frequently required for appropriate preoperative imaging evaluations of a patient with suspected HC. For tumor staging, ultrasonography, contrast-enhanced multidetector-row CT (MDCT) and cholangiography were typically performed. If necessary, some patients were selected for magnetic resonance imaging (MRI) and positron emission tomography (PET) scans, and patients with imaging signs of distant metastasis (M1), including the liver, peritoneum and lymph nodes, were deemed unresectable.

The Bismuth-Corlette classification was applied to patients with imaging modalities that confirmed
HC. A multidisciplinary team (MDT) meeting was held to evaluate the probability of RO resection. In this meeting, the involvement of the vasculature around the hilar, the longitudinal and radial extension of the tumor, and the presence of radiologically proven metastases were comprehensively discussed by hepatobiliary surgeons, consultant oncologists, hepatologists, radiologists and specialist nurses. All tumors $(\mathrm{HC})$ that were shown to be potentially resectable were later subjected to laparoscopic exploration and curative resection.

Laboratory examinations included measurements of CEA, Ca19-9 and liver function tests. Not all patients with overt jaundice required biliary drainage; the decision was balanced with the benefit and increased risk of perioperative complications and was made in a multidisciplinary setting. Any required presurgical biliary drainage was performed only into the reserved liver remnant.

Surgery was not continued in cases of the unexpected presence of metastatic disease. However, if the planned operation was not considered too risky and was expected to likely improve patient survival and quality of life, even if positive periaortic nodes were detected on frozen sections during the operation, resection was also undertaken according to the plan.

\section{Technical aspects of the surgical approach}

After general anesthesia, patients were placed in the supine reverse Trendelenburg position with the possibility to be tilted during the operation. The patient was firmly secured to the table. Both arms were abducted at a 90-degree angle to the body, and both legs were kept apart, slightly bent, and abducted to provide stability to the patient. All patient pressure areas were protected by soft devices. The surgeon stood either at the patient's the right side or between the patient's legs depending on the technical requirements during the operation. Pneumoperitoneum was established by a port positioned below the umbilicus and maintained at $<13 \mathrm{~mm} \mathrm{Hg}$. Staging laparoscopy was achieved as previously reported [32]. For patients with no signs of metastatic disease, the remaining ports were placed as shown in Figure 1.

Intraoperative ultrasonography was used to check and evaluate possible intrahepatic metastasis at the beginning of the laparoscopic $\mathrm{HC}$ resection surgery. 


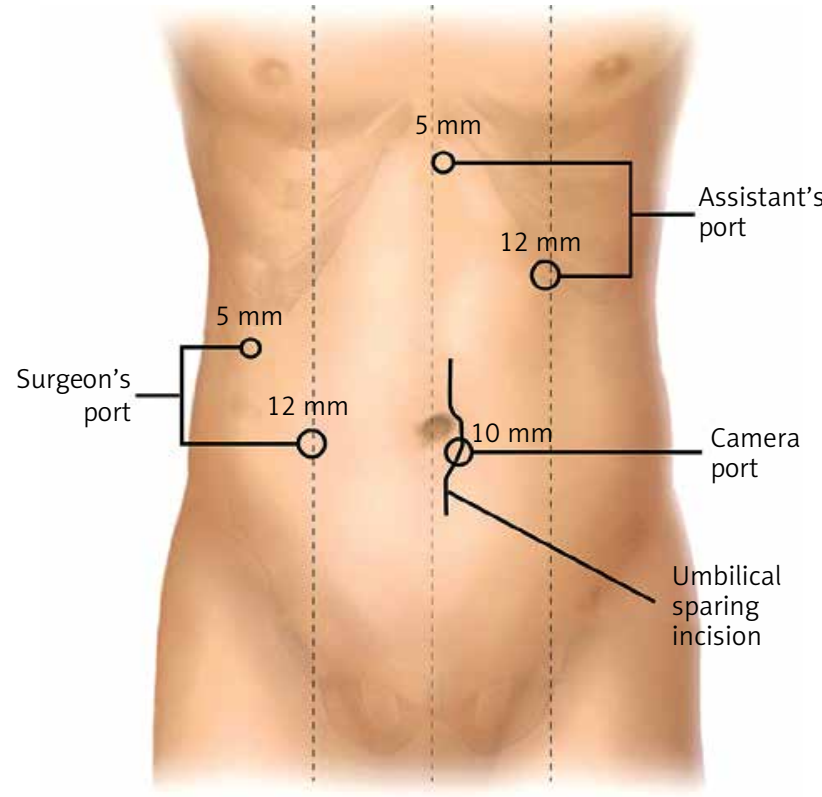

Figure 1. Trocar placement in laparoscopic radical resection for Bismuth type IV HC
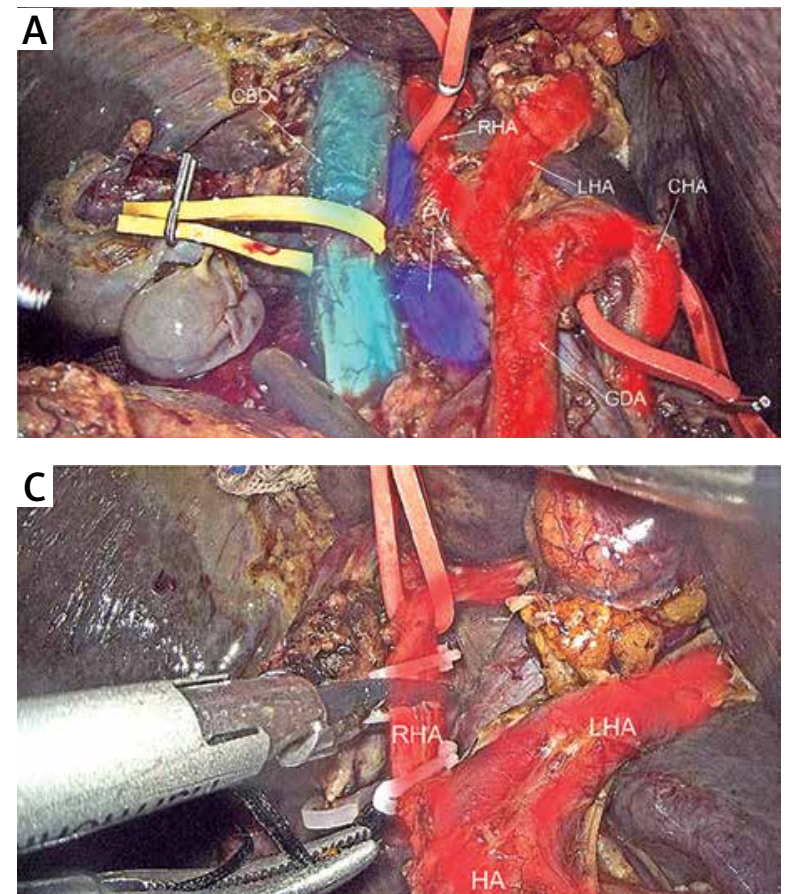

Then, the Kocher maneuver was used to mobilize the duodenum and dissect the regional No. 16 a2/ b1 lymph nodes. The lymph nodes scattered along the entire length of the hepatic artery from the celiac axis to the level of its bifurcation and the length of the right or left hepatic arteries as well as the pericholedochal, peri/retroportal and retropancreatic lymph nodes were routinely dissected. A circumferential excision of the hepatoduodenal ligament was performed, and the entire lymph nodal tissue was removed en bloc (Figure 2 A). The distal common bile duct at the upper border of the pancreatic head was subsequently transected for frozen biopsy (Figure 2 B). Subsequently, the inflow of the left or right liver was occluded and divided (Figures 2 C, D). The right/left lobes and caudate lobe were completely mobilized and excised en bloc (Figures 2 E, F). A specimen retrieval bag was used to remove the specimen through an additional 5 - to $10-\mathrm{cm}$ umbilical sparing median incision. This incision was closed immediately, and the pneumoperitoneum was re-
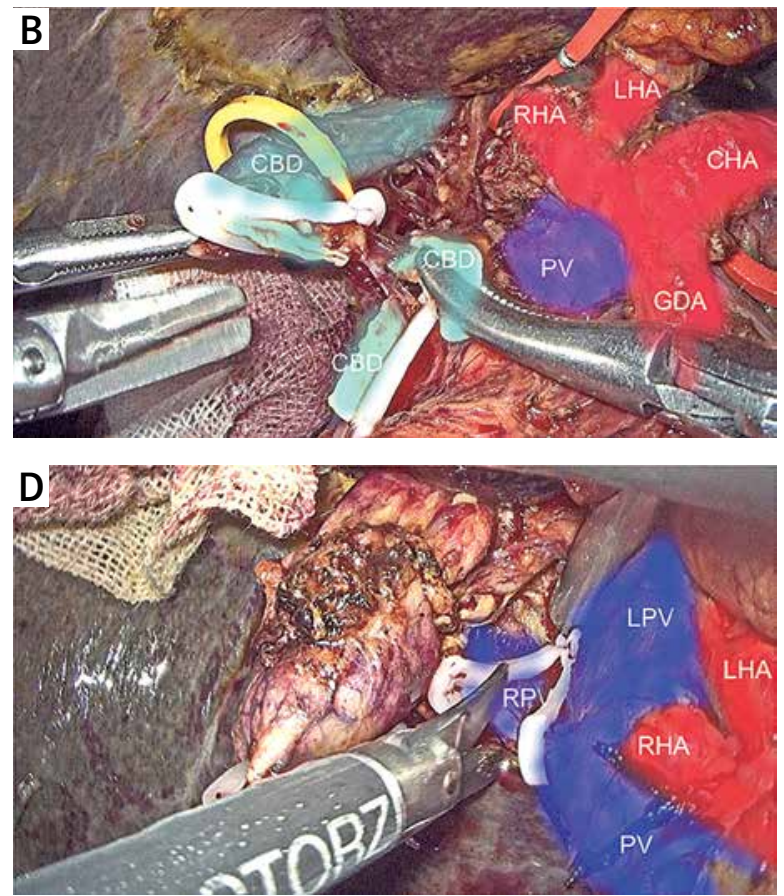

Figure 2. Laparoscopic right hemihepatectomy with caudate lobectomy, hepatoduodenal lymphadenectomy and bilioenteric anastomosis to achieve radical resection for patients with Bismuth IV HC. A - Skeletonization of the vasculature and dissection of lymph nodes in the suprapancreatic area. $\mathbf{B}-$ The distal common bile duct was transected with scissors at the upper border of the pancreas, and the specimen was obtained for frozen biopsy to confirm RO resection. C, D - The right hepatic artery (C) and portal vein (D) were occluded and divided

$C B D$ - common bile duct, CHA - common hepatic artery, PV - portal vein, RHA - right hepatic artery, LHA - left hepatic artery, HA - hepatic artery, $R P V$ - right portal vein, $L P V$ - left portal vein, GDA - gastroduodenal artery. 

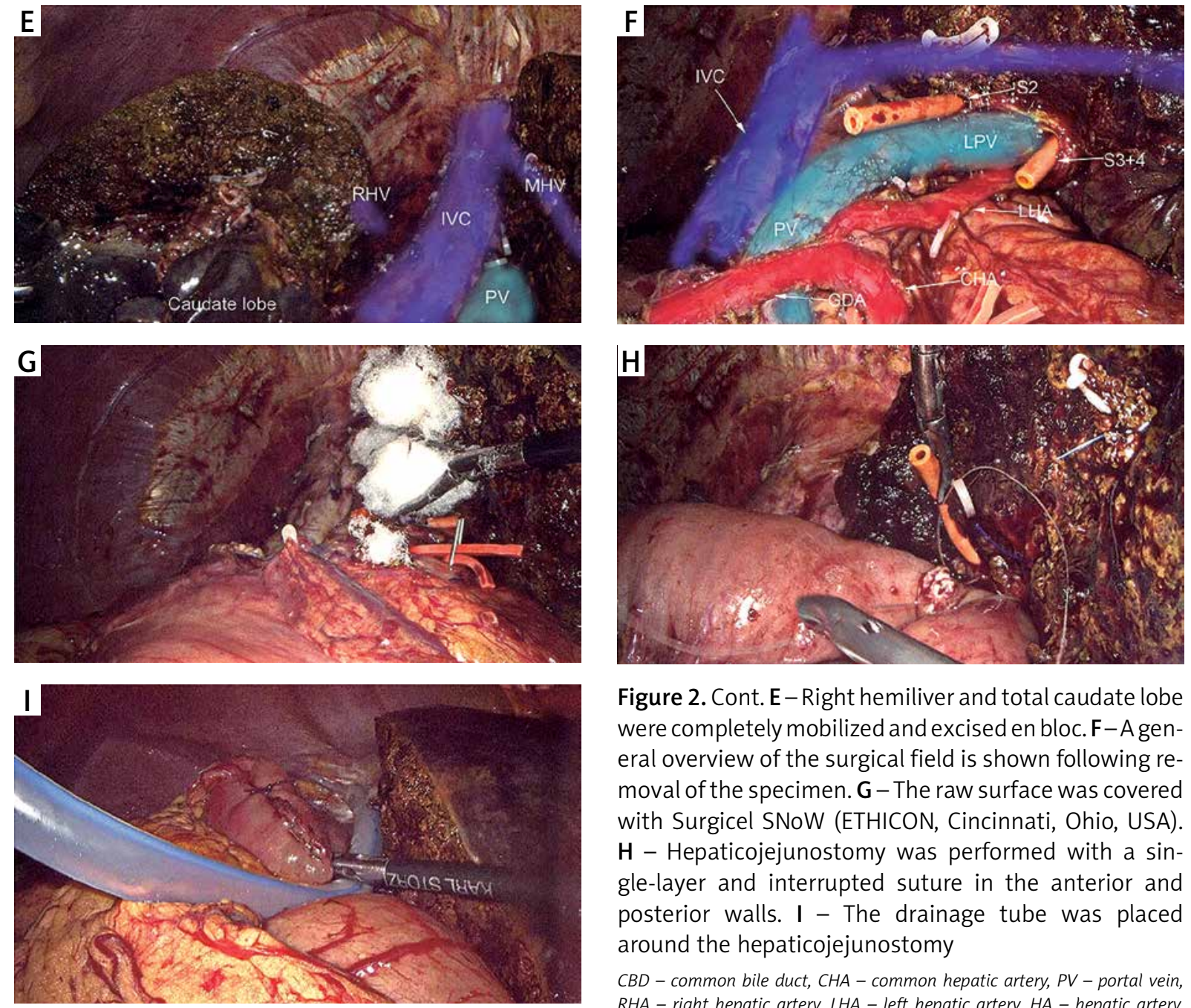

Figure 2. Cont. E-Right hemiliver and total caudate lobe were completely mobilized and excised en bloc. F-Ageneral overview of the surgical field is shown following removal of the specimen. $\mathbf{G}$ - The raw surface was covered with Surgicel SNoW (ETHICON, Cincinnati, Ohio, USA). H - Hepaticojejunostomy was performed with a single-layer and interrupted suture in the anterior and posterior walls. I - The drainage tube was placed around the hepaticojejunostomy

$C B D$ - common bile duct, CHA - common hepatic artery, PV - portal vein, RHA - right hepatic artery, $L H A$ - left hepatic artery, HA - hepatic artery, $R P V$ - right portal vein, $L P V$ - left portal vein, GDA - gastroduodenal artery.

sumed. Saline was used to irrigate the surgical field, and then bile leakage and bleeding were checked. After achieving hemostasis and biliostasis, the raw surface was covered with Surgicel SNoW (ETHICON, Cincinnati, Ohio, USA) (Figure $2 \mathrm{G}$ ). The biliary flow into the alimentary system was reconstructed by a Roux-en-Y bilioenteric anastomosis. A single-layer and interrupted bilioenteric anastomosis was performed to restore the biliary flow (Figure $2 \mathrm{H}$ ). At the end of the procedure, the surgical field was systemically explored for potential complications. Then, a drainage tube was placed around the hepaticojejunostomy (Figure $2 \mathrm{l}$ ).

For liver transection, a crush/clamp technique using a laparoscopic Harmonic Scalpel and bipolar forceps was adopted, along with associated saline pulses and aspiration. This process was performed

with the alternate Pringle maneuver with decreased central venous pressure as previously described [39]. For the Pringle maneuver, cotton tape is used to pass around the hepatic pedicle. Then, a dedicated 5-mm port was created around the umbilicus that did not obstruct the field, and a tubular drain was applied to snugly attach the cotton tape with the help of a grasper. Another technique that allows for direct encircling of the hepatic pedicle was also utilized as necessary. The Pringle maneuver can be safely performed for 15 to 20 min and could be repeated many times during the whole procedure, with 5- to 10-minute perfusion between each maneuver.

\section{Data collection and statistical analysis}

Perioperative results included the laboratory examination, Bismuth-Corlette classification, blood 


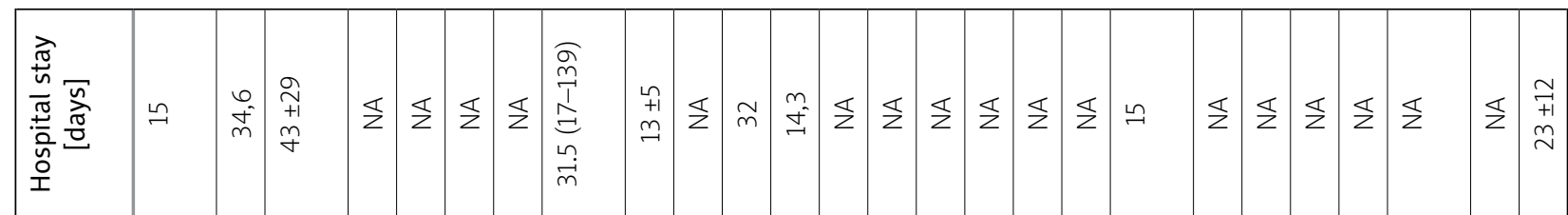

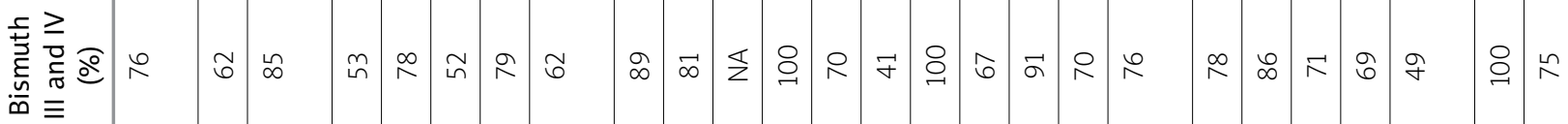

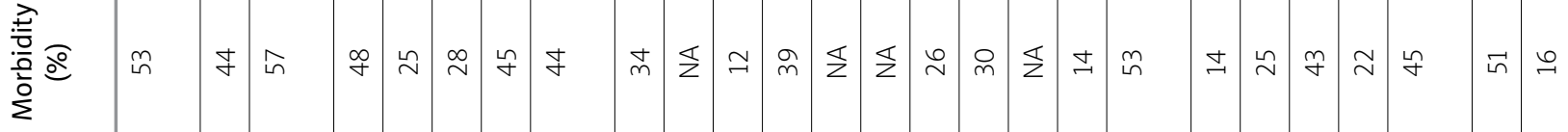

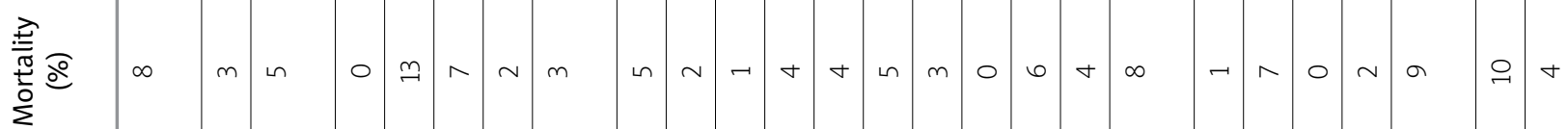

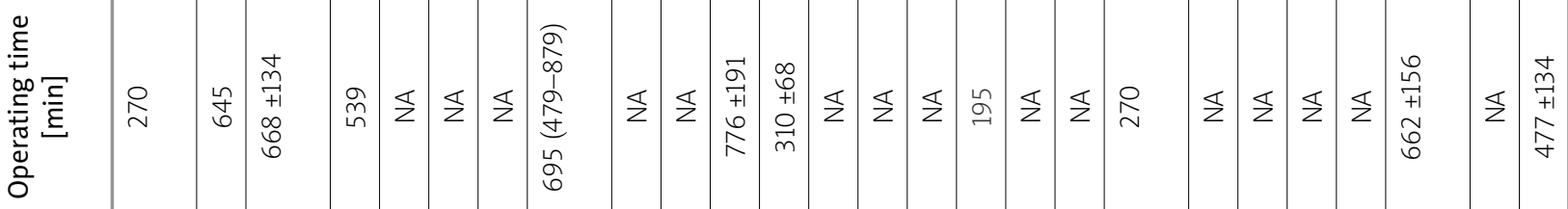

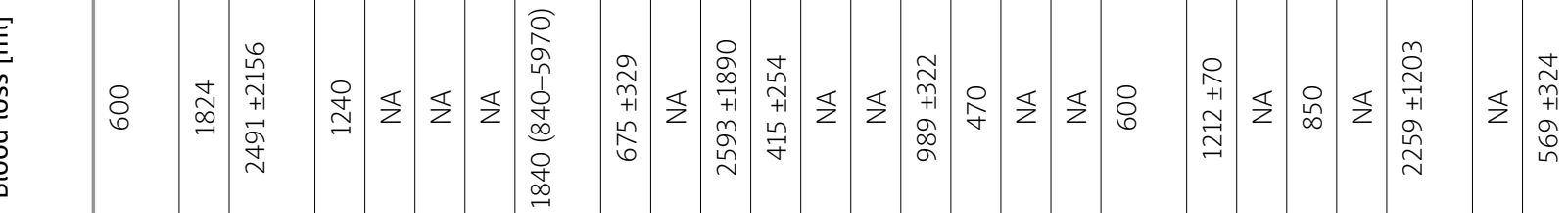

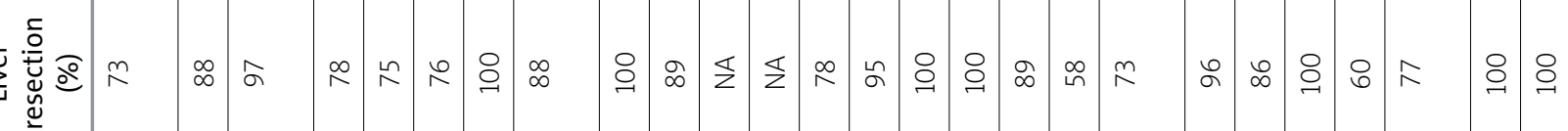

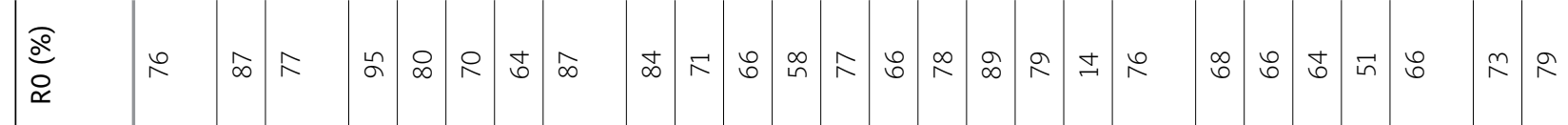

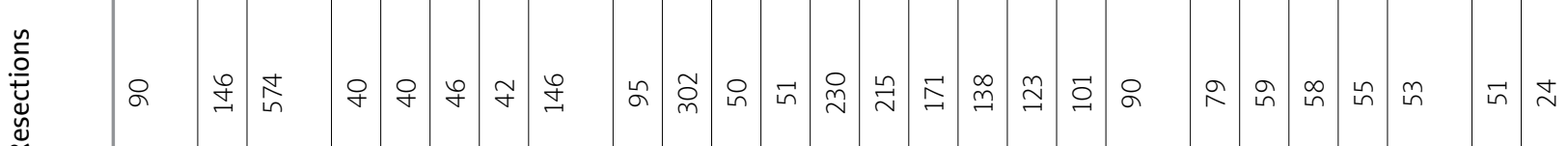

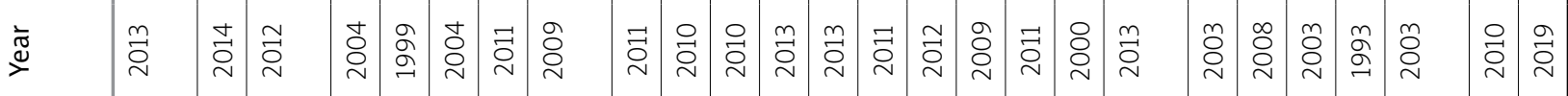

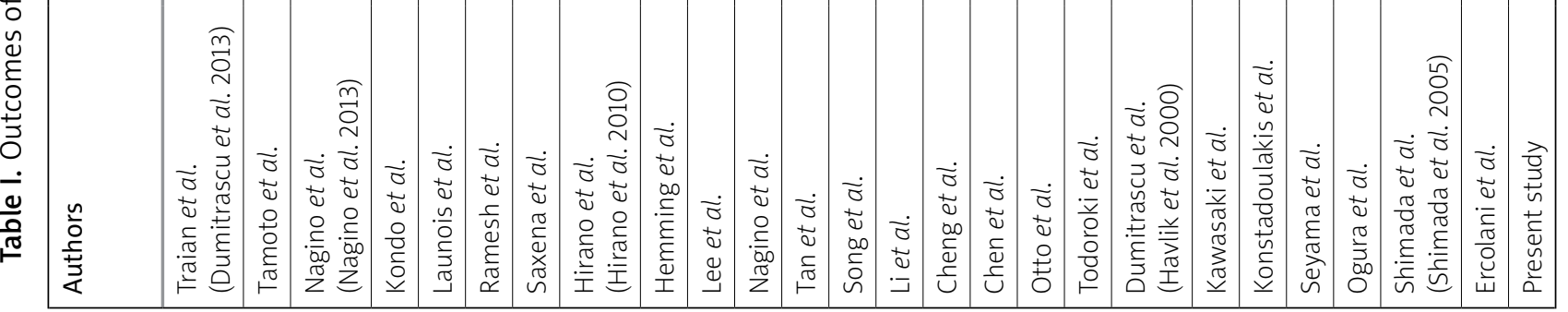


loss, operative time, need for blood transfusion, biopsy of resection margins, 90-day mortality, postoperative morbidity, and the duration and cost of hospital stay. Operative time was determined from the beginning of pneumoperitoneum to the last port incision closure, including the instrumental preparation time. Postoperative morbidity was described according to the Clavien-Dindo classification. Patients were followed up for tumor condition and long-term prognosis through telephone interviews or individual clinic visits.

Additionally, a systematic search of all English literature was performed in PubMed and EMBASE based on the following search strategy: "hilar cholangiocarcinoma OR Klatskin tumor". The retrieved articles were manually checked one by one to exclude studies without original data and that did not focus on curative surgical resection. Studies and series reports that included fewer than 40 resections were not included. Finally, the results of radical resection of patients with $\mathrm{HC}$ from 25 studies published from 1993 to 2014 were extracted and are detailed in Table I. Publicly available data about the long-term outcomes of HC patients who underwent curative-intent resection were obtained from 10 U.S. academic medical centers [40]. The Kaplan-Meier method was used for survival analysis. All statistical analyses were processed using $\mathrm{R}$ software (version 3.2.2).

\section{Results}

\section{Preoperative characteristics (Table II)}

During the 45-month study period, 32 patients underwent radical laparoscopic resection surgery for HC. The detailed baseline characteristics of the patients are detailed in Table I. The female-to-male ratio was $11: 21$, and the median (range) age of the patients was $60.6(38.8-76.7)$ years. The body mass index was $22.6(18.4-29.1) \mathrm{kg} / \mathrm{m}^{2} ; 23$ (71.8\%) patients were in the normal range. The preoperative American Society of Anesthesiologists (ASA) score, which assesses the physical status before surgery, was 1 for most (71.8\%) patients. Twenty-two (68.7\%) patients had preoperative jaundice, and 14 (43.8\%) patients with irreversible severe jaundice underwent preoperative biliary drainage. A percutaneous transhepatic biliary drain was inserted in 12 (37.6\%) patients, and endoscopic nasogastric drainage was performed in 2 (6.2\%) patients. Based on
Table II. Patients' preoperative characteristics

\begin{tabular}{|c|c|}
\hline Variables & Value \\
\hline \multicolumn{2}{|l|}{ Gender: } \\
\hline Male & $21 / 32(65.6 \%)$ \\
\hline Female & $11 / 32(34.4 \%)$ \\
\hline Age (range) [years] & $60.6(38.8-76.7)$ \\
\hline \multicolumn{2}{|l|}{ BMI [kg/m²]: } \\
\hline$<18.5$ & $1 / 32(3.1 \%)$ \\
\hline $18.5-24.9$ & $23 / 32(71.8 \%)$ \\
\hline $25-29.9$ & $8 / 32(25.0 \%)$ \\
\hline$>30$ & 0/32 (0\%) \\
\hline \multicolumn{2}{|l|}{ ASA: } \\
\hline 1 & 23/32 (71.8\%) \\
\hline 2 & $7 / 32(21.8 \%)$ \\
\hline 3 & $2 / 32(6.4 \%)$ \\
\hline \multicolumn{2}{|l|}{ Biliary drainage: } \\
\hline No & $18 / 32(56.2 \%)$ \\
\hline ERCP & $2 / 32(6.2 \%)$ \\
\hline PTCD & $12 / 32(37.6 \%)$ \\
\hline Comorbidity & $10 / 32(31.2 \%)$ \\
\hline \multicolumn{2}{|l|}{ Child classification: } \\
\hline$A$ & $7 / 32(21.8 \%)$ \\
\hline $\mathrm{B}$ & $25 / 32(78.2 \%)$ \\
\hline \multicolumn{2}{|l|}{ Bismuth type: } \\
\hline I & 0/32 (0\%) \\
\hline$\|$ & 6/32 (18.7\%) \\
\hline IIla & 4/32 (12.5\%) \\
\hline$\| l l b$ & $8 / 32(25.0 \%)$ \\
\hline IV & $14 / 32(43.8 \%)$ \\
\hline \multicolumn{2}{|l|}{ Laboratory tests: } \\
\hline $\mathrm{TBIL}[\mu \mathrm{mol} / \mathrm{I}]$ & $169.04 \pm 127.23$ \\
\hline $\mathrm{DBIL}[\mu \mathrm{mol} / \mathrm{I}]$ & $102.63 \pm 90.44$ \\
\hline $\mathrm{ALB}[\mathrm{g} / \mathrm{l}]$ & $38.33 \pm 9.19$ \\
\hline AST [U/I] & $105.74 \pm 92.11$ \\
\hline $\mathrm{ALT}[\mathrm{U} / \mathrm{I}]$ & $138.08 \pm 167.6$ \\
\hline $\mathrm{CEA}[\mathrm{kU} / \mathrm{l}]$ & $9.71(0.91,126.26)$ \\
\hline CA199 [kU/l] & $3345.64(1.93,46428.84)$ \\
\hline
\end{tabular}

$A L B$ - albumin, ALT - alanine aminotransferase, AST - aspartate aminotransferase, $B M I$ - body mass index, PTCD - percutaneous transhepatic catheter drainage, $E R C P$ - endoscopic retrograde cholangio-pancreatography 
the preoperative imaging findings and MDT discussion, Bismuth types I, II, IIIa, IIIb, and IV cholangiocarcinoma were diagnosed in 0, 6 (18.7\%), 4 (12.5\%), 8 (25.0\%), and $14(43.8 \%)$ patients, respectively. The preoperative total bilirubin, direct bilirubin, alanine aminotransferase (ALT), aspartate aminotransferase (AST), ALB, CEA, and CA 199 levels are detailed in Table II.

Table III. Intraoperative data

\begin{tabular}{|c|c|}
\hline Variables & Value \\
\hline Surgery type: & $N=32$ \\
\hline Laparoscopic exploration & $3 / 32(9.3 \%)$ \\
\hline Laparoscopic radical resection & $24 / 32(75.0 \%)$ \\
\hline $\begin{array}{l}\text { Conversion from laparoscopy to } \\
\text { laparotomy }\end{array}$ & $5 / 32(15.7 \%)$ \\
\hline Laparoscopic radical resection: & $N=24$ \\
\hline Operating time [min] & $476.95 \pm 133.89$ \\
\hline Estimated blood loss [ml] & $568.75 \pm 324.01$ \\
\hline \multicolumn{2}{|l|}{ Transfusion requirement: } \\
\hline Plasma (range) [ml] & $127(0-400)$ \\
\hline Red cell (range) [U] & $2.5(0-10.0)$ \\
\hline \multicolumn{2}{|l|}{ Surgical radicality: } \\
\hline $\mathrm{RO}$ & 19/24 (79.1\%) \\
\hline $\mathrm{R} 1$ & $3 / 24(12.5 \%)$ \\
\hline $\mathrm{R} 2$ & $2 / 24(8.4 \%)$ \\
\hline \multicolumn{2}{|l|}{ Type of hepatectomy: } \\
\hline Right hepatectomy & $7 / 24(29.1 \%)$ \\
\hline Left hepatectomy & $3 / 24(12.5 \%)$ \\
\hline Hepatectomy with caudate lobe & $14 / 24(58.4 \%)$ \\
\hline \multicolumn{2}{|l|}{ Bismuth type: } \\
\hline । & $0 / 24(0 \%)$ \\
\hline$\|$ & $2 / 24(8.4 \%)$ \\
\hline IIIa & $4 / 24(16.6 \%)$ \\
\hline$I I I b$ & $5 / 24(20.8 \%)$ \\
\hline IV & $13 / 24(54.2 \%)$ \\
\hline Tumor size $[\mathrm{cm}]$ & $3.076 \pm 0.38$ \\
\hline Lymph node received & $8.93 \pm 5.26$ \\
\hline
\end{tabular}

$R O$ - negative margin, $R 1$ - microscopic positive margin, $R 2$ - macroscopic residual tumor resection.

\section{Intraoperative parameters (Table III)}

Among the 32 included patients, laparoscopic surgery with radical resection was ultimately performed in 24 (75.0\%) patients. Three (9.3\%) patients were found to be unresectable at the time of preliminary exploration, among whom 2 had peritoneal carcinomatosis and 1 had extensive duct spread with evidence of contralateral hepatic artery involvement. Five (15.7\%) patients converted from laparoscopy to laparotomy due to accidental severe bleeding, anatomical variation or tumors invading the proximal trunk of the portal vein. Laparoscopic hemihepatectomy with caudate lobectomy was performed in 14 (65.6\%) patients, with the right lobe resected in 6 patients and the left lobe resected in 8 . The operation time and blood loss were $476.95 \pm 133.89 \mathrm{~min}$ and $568.75 \pm 324.01 \mathrm{ml}$, respectively. A Pringle maneuver was performed for 21 (77.8\%) patients, with a median time (range) of 30 (15-60) min. A negative margin (R0) was achieved in 19 (79.1\%) patients. Three $(12.5 \%)$ patients were identified with a microscopic positive margin (R1). Because of macrovascular infiltration, 2 (8.4\%) patients underwent macroscopic residual tumor resection (R2). After surgery, Bismuth types II, IIIa, IIIb, and IV cholangiocarcinoma were diagnosed in $2(8.4 \%), 4(16.6 \%)$, 5 (20.8\%), and 13 (54.2\%) patients, respectively. The tumor diameter was $3.076 \pm 0.38 \mathrm{~cm}$, and the number of dissected lymph nodes was $8.93 \pm 5.26$. All of the abovementioned parameters are summarized in Table III.

\section{Postoperative and oncological outcomes (Table IV)}

The length of postoperative stay was $23.3 \pm 11.7$ days, including patients who suffered complications. There was 1 (4.1\%) patient death within 30 days after surgery; the patient suffered serious intra-abdominal bleeding and consequent disseminated intravascular coagulation (DIC). The pathologic results showed that cholangiocarcinoma and cholangiocarcinoma combined with mucinous adenocarcinoma were confirmed in $21(87.5 \%)$ and $3(12.5 \%)$ patients, respectively. Postoperative complications were stratified according to the Clavien-Dindo classification. Grade I-II complications occurred in 15 (62.5\%) patients. Severe morbidity was defined as grade III-V, which occurred in $4(16.6 \%)$ patients. The hospital expenditure was USD $14348 \pm 4779$. After a median 
(range) follow-up of 9 (1.5-45.1) months, 16 (66.7\%) patients were alive without any sign of recurrence. Liver recurrence occurred in 3 (12.5\%) patients, retropancreatic lymph node metastasis in 1 (4.1\%) patient and multisite metastasis in 1 (4.1\%) patient, including the liver and peritoneum (Table IV).

\section{Comparisons with conventional surgery results (real-world evidence)}

The extent of surgical resection for $\mathrm{HC}$ with the aim of curative treatment is already controversial. To date, numerous studies have published the oncological outcomes of the radical resection of $\mathrm{HC}$ though open surgery. These studies included a large number of patients with long-term oncological follow-up. The final results have tended towards stability and can reflect the actual effect of conventional open surgery. Thus, the outcomes of surgical resection from 25 studies published during the period 19932014 were extracted and compared with our results in the present study in Table I. Additionally, we further compared the long-term outcomes with publicly available data obtained from 10 U.S. academic medical centers [40]. As shown in Figure 3, HC patients who underwent curative-intent resection though open surgery had actuarial 3-year overall survival (OS) and recurrence-free survival (RFS) rates of $36.2 \%$ and $27.8 \%$, respectively. However, the actuarial 3-year OS and RFS for laparoscopic surgery in the present study were $49.1 \%$ and $47.0 \%$, respectively.

\section{Discussion}

Due to the overt benefits over open surgery, including decreased wound-related complications, early ambulation, quicker recovery time and much smaller scarring, laparoscopic surgery has been routinely used in the management of benign and malignant disease. Over the past decades, many kinds of laparoscopic surgery have evolved; at first unsuccessful, subsequent but still early procedures began showing some benefit, and now they are becoming the gold standard form of surgery. These promising results have encouraged surgeons to further explore the application of laparoscopic techniques in complex surgery. In fact, a similar process can also be observed in laparoscopic pancreaticoduodenectomy (LPD) [41] and laparoscopic hepatectomy [42], which was initially controversial but has been gradually accepted. For example, LPD was once regarded as the Mt. Everest of general surgery [43]. Even conventional laparotomy is considered hard to perform, let alone laparoscopic resection. However, after nearly 25 years of endeavor, recently published studies have finally confirmed that LPD is a safe and feasible procedure from a technical standpoint, with acceptable rates of mortality and morbidity [44, 45]. Wang et al. retrospectively analyzed 1029 consecutive patients who had undergone LPD in China and found that median operation time, major complications, and conversion rate improved significantly with sur-

Table IV. Patients' postoperative parameters and pathological results

\begin{tabular}{|c|c|}
\hline Variables & Value \\
\hline Postoperative hospital stay [days] & $23.3 \pm 11.7$ \\
\hline \multicolumn{2}{|l|}{ Complications, $n /$ total (\%): } \\
\hline No & $5 / 24(20.8 \%)$ \\
\hline Yes & $19 / 24(79.2 \%)$ \\
\hline \multicolumn{2}{|c|}{ Clavien-Dindo grading system, $n /$ total (\%): } \\
\hline$|-| \mid:$ & $15 / 24(62.5 \%)$ \\
\hline Pleural effusion & $8 / 24(33.3 \%)$ \\
\hline Abdominal infection & $6 / 24(25.0 \%)$ \\
\hline Pulmonary infection & $6 / 24(25.0 \%)$ \\
\hline Incisional infection & $1 / 24(4.1 \%)$ \\
\hline Gastroparesis & $4 / 24(16.6 \%)$ \\
\hline Bile leakage & $4 / 24(16.6 \%)$ \\
\hline III-IV: & $4 / 24(16.6 \%)$ \\
\hline Intra-abdominal bleeding & $1 / 24(4.1 \%)$ \\
\hline Pleural effusion & $1 / 24(4.1 \%)$ \\
\hline $\mathrm{DIC}$ & $1 / 24(4.1 \%)$ \\
\hline Pulmonary infection & $1 / 24(4.1 \%)$ \\
\hline Gastrointestinal hemorrhage & $1 / 24(4.1 \%)$ \\
\hline 90-day mortality, n/total (\%) & $1 / 24(4.1 \%)$ \\
\hline \multicolumn{2}{|l|}{ Pathology, n/total (\%): } \\
\hline Cholangiocarcinoma & $21 / 24(87.5 \%)$ \\
\hline Cholangiocarcinoma + MA & $3 / 24(12.5 \%)$ \\
\hline Overall cost $[\$]$ & $14348 \pm 4779$ \\
\hline Follow-up, median [months] & 9.0 \\
\hline Recurrence free, median [months]: & 31.5 \\
\hline Recurrence site: & $5 / 24(20.8 \%)$ \\
\hline Liver & $3(12.5 \%)$ \\
\hline Lymph node & $1(4.1 \%)$ \\
\hline Multi-site & $1(4.1 \%)$ \\
\hline
\end{tabular}




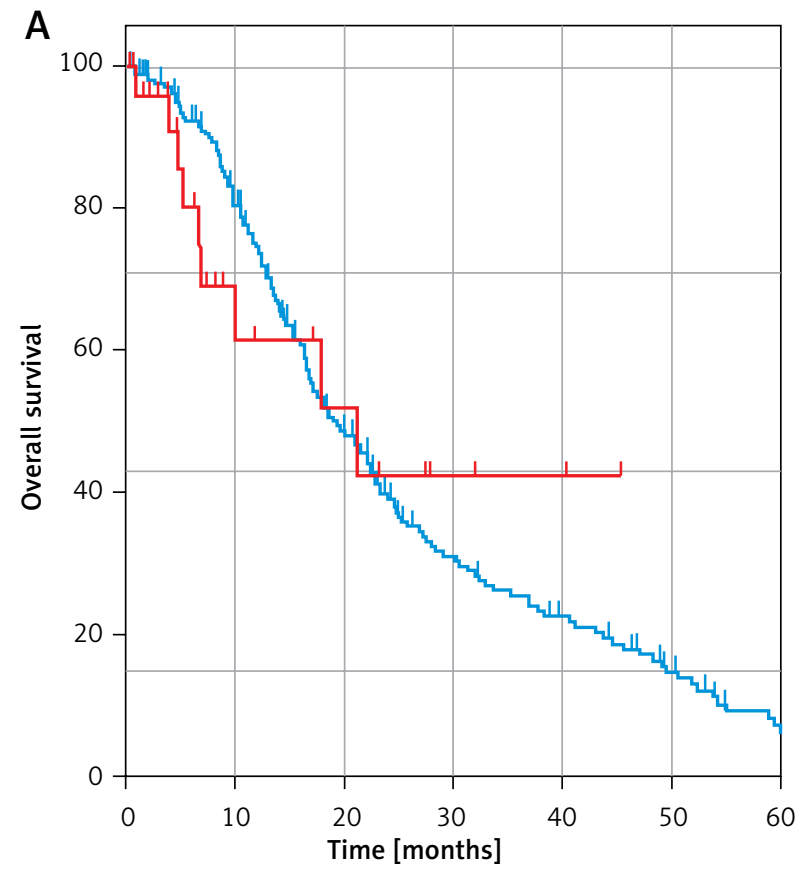

Open surgery _Laparoscopic surgery

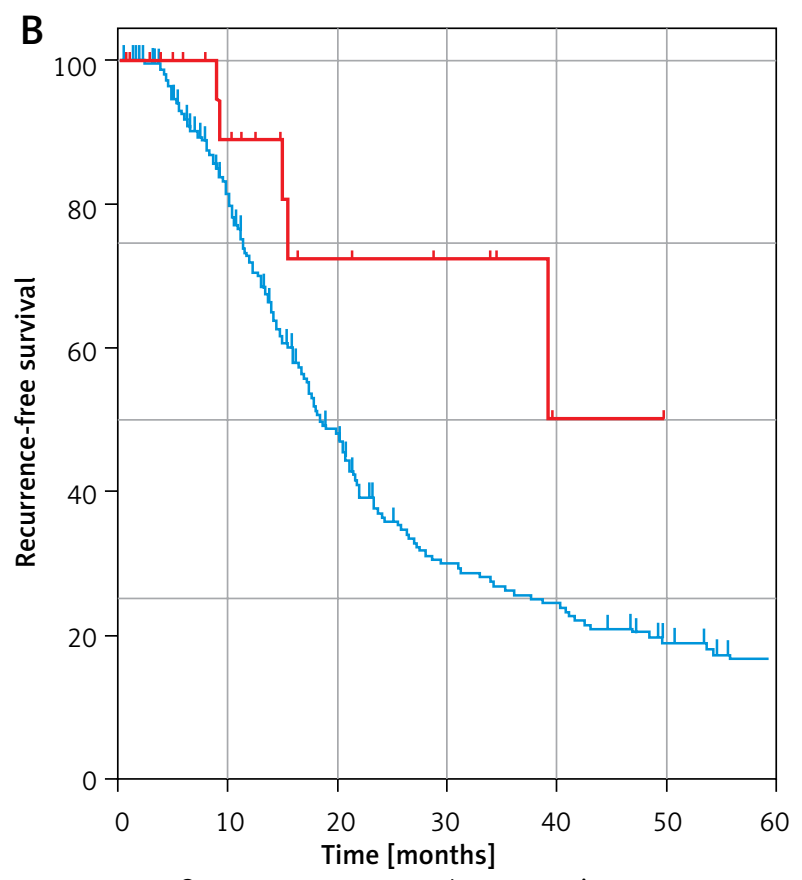

- Open surgery Laparoscopic surgery

Figure 3. Kaplan-Meier curve of open surgery (blue) and laparoscopic surgery (red) for patients with HC who underwent curative-intent resection. A - Kaplan-Meier curve demonstrating actuarial overall survival. B - Kaplan-Meier curve demonstrating recurrence-free survival

geon's experience [44]. More importantly, as one of the most complicated operations, LPD has a learning curve that is significantly longer than expected. As demonstrated in a previous study, only after completing at least 140 cases can competence for LPD be achieved [44]. Additionally, another study published at the same time reported their experience from 500 patients in a single center. This study revealed that LPD is a feasible choice for selected patients [45]. After overcoming the learning curve, perioperative outcomes and the prognosis of LPD can be significantly optimized [45]. Taken together, the abovementioned studies highlight that even the Mt. Everest of general surgery can be climbed with the accumulation of surgical experience and the development of laparoscopic techniques. At present, LPD, an incredibly difficult operation, has been transformed into routine surgery in high volume hospitals.

If LPD is the Mt. Everest of general surgery, laparoscopic radical resection of $\mathrm{HC}$ is the brightest jewel at the top of the mountain. As the greatest challenge for hepatobiliary surgeons, the exploration of minimally invasive surgery for $\mathrm{HC}$ was started by Giulianotti et al. in 2010 [46], 16 years after the invention of LPD. This in itself reflects the extremely high technical demands of laparoscopic radical resection for HC. HC is completely different from other cholangiocarcinomas in terms of the therapeutic strategy and prognosis $[1,47]$. This is because HC arises from the biliary confluence or the left or right hepatic duct, which are close to vascular structures. There are variable, complex and intimate relationships between the biliary and vascular structures at this location. Meanwhile, the tumor properties allow $\mathrm{HC}$ to invade vascular structures, infiltrate adjacent liver tissues, spread along the bile ducts and metastasize to the lymph nodes and distant organs [4850]. Undoubtedly, margin negative (RO) resection is the most effective treatment and probably the only approach that provides a chance of cure. However, radical resection with a negative margin is extremely challenging. The standard operations for resectable $\mathrm{HC}$ include extended liver resections in conjunction with extrahepatic bile duct resection, hepatoduodenal lymphadenectomy, caudate lobectomy and bilioenteric anastomosis [3, 10]. Increasing evidence has demonstrated that this "aggressive" surgical strategy, although technically challenging, has increased the rate of curative resection and long-term survival. Unfortunately, $29-40 \%$ of HC patients had locally 
advanced or metastatic disease at initial presentation, making them ineligible for surgical resection $[13,51]$. Moreover, at the time of the curative-intent operation, only $50-60 \%$ of patients have been ultimately found to be resectable [51, 52]. In the current study, all enrolled patients were highly selected and considered suitable for curative-intent surgery. In contrast to what was observed in the above studies, only $9.3 \%(3 / 32)$ of the HC patients were found to be unresectable at the time of surgical exploration in the present study. This result may, in part, be due to the comprehensive use of various imaging techniques (thinly sliced CT, MRI, and USG) to evaluate tumor resectability and the selection of patients who could undergo laparoscopic surgery by the MDT approach.

With the accumulated experience of laparoscopic surgery and the development of laparoscopic devices, an increasing number of studies have reported the safety and feasibility of laparoscopic extended hemihepatectomy [53, 54], laparoscopic caudate lobectomy [55, 56], laparoscopic hepatoduodenal lymphadenectomy and bilioenteric anastomosis [44, 45]. Thus, from a technical perspective, it is obvious that the essential surgical techniques for laparoscopic resection of $\mathrm{HC}$ have become more reliable and diverse. To date, however, very few experienced surgeons have attempted to challenge the laparoscopic approach for the radical resection of $\mathrm{HC}$. According to a systematic review published recently, only 21 studies have been published, reporting on a total of 142 minimally invasive procedures for HC, among which 1 hybrid, 59 robot-assisted and 82 laparoscopic procedures were included [58]. Margin-negative resection (R0) was achieved in almost $80 \%$ of patients, and only $4.9 \%$ of patients converted from laparoscopy to laparotomy. Furthermore, the total of 30 complications and 4 deaths among 126 patients suggests a postoperative morbidity rate of $24 \%$ and an overall 90 -day mortality rate of $3 \%$. These results indicated that laparoscopic radical resection of $\mathrm{HC}$ is technically feasible for highly selected patients in the hands of experienced surgeons. In the current study, an R0 resection with adequate lymphadenectomy was achieved in $79.1 \%$ of patients. Additionally, the postoperative morbidity rate and overall 90-day mortality rate were $16.6 \%$ and $4.1 \%$, respectively. Obviously, for major perioperative indicators, such as $\mathrm{RO}$ resection rate, 90-day mortality rate, and postoperative morbidity rate, the results from our current study and the systematic review [57] are very similar.

However, the rate of conversion to laparotomy in laparoscopic hepatectomy ranged from $9 \%$ to $42 \%$ [58], and even in laparoscopic cholecystectomy, the rate of conversion from laparoscopy to laparotomy remains between $5 \%$ and $10 \%$ [59]. Additionally, at the initial and exploratory stages, the conversion rate of LPD in high volume centers can reach $17.3 \%$. Even when surgeons overcome the learning curve (performing more than 104 cases), the average conversion rate can only be reduced to $5.9 \%$, which is higher than the result in the systematic review (4.9\%). In the present study, $15.7 \%$ of patients converted from laparoscopy to laparotomy due to accidental severe bleeding, anatomical variation and tumors invading the proximal trunk of the portal vein, which is similar to the result for LPD (17.3\%) in its infancy. Outcomes of open surgery for $\mathrm{HC}$ have been reported by several multicenter studies and single-institutional series, and these larger case series are detailed in Table I. The morbidity and mortality of open surgery in patients with $\mathrm{HC}$ are notoriously high and have been reported to reach $18 \%$ and $68 \%$, respectively. Additionally, publicly available data on the long-term outcomes of HC patients who underwent curative-intent surgery, obtained from 10 U.S. medical centers, were comparatively analyzed in this study. As shown in Figure 3, HC patients who underwent curative-intent resection though open surgery had an actuarial 3 -year OS and RFS of $36.2 \%$ and $27.8 \%$, respectively. However, the actuarial 3-year OS and RFS for laparoscopic surgery in the present study were $49.1 \%$ and $47.0 \%$, respectively.

These comparisons with the literature (Table I) demonstrate a benefit of laparoscopic radical resection for HC compared with open surgery. However, the abovementioned findings should be interpreted with extreme caution. These preliminary results may not be truly representative of current practice and are very likely to be influenced by strict patient selection, which is also a limitation of this current study. Therefore, the results cannot be widely reproduced and the use of laparoscopic radical resection of $\mathrm{HC}$ for this specific patient population should be limited to experienced centers only.

To the best of our knowledge, the current study is one of the largest series regarding the feasibility and safety of laparoscopic resection for HC. Combining the results from previously published articles and 
those of the present study, it is safe to say that the laparoscopic radical resection of $\mathrm{HC}$ is feasible from a technical point of view but is still in its initial stages. However, before the laparoscopic approach for patients with $\mathrm{HC}$ becomes routinely implemented, the questions that need to be solved involve not only the safety and technical feasibility but also oncological concerns. For example, is there any increase in the incidence of port-site metastases? Can the longterm oncological outcomes be compared to those of an open procedure?

In the present study, throughout a median (range) follow-up of 9 (1.5-45.1) months, no patients with $\mathrm{HC}$ who were treated by laparoscopic radical resection suffered recurrence at the port site. Similar to what we observed in this series, earlier studies also indicated that implanted metastasis or port site recurrence (PSR) did not occur in any patients during a median follow-up of 48 and 60 months, respectively $[31,35]$. Previous studies have also demonstrated that the median time of PSR is 6 months [60]. Thus, it is reasonable to assume that laparoscopic radical resection for $\mathrm{HC}$ does not increase the incidence of PSR. Combining both the former experimental studies and our own observations, the effect of pneumoperitoneum alone on PSR and implanted metastasis is almost negligible. However, due to the possibility of cell exfoliation during tumor handling, which may result in the dissemination or direct contamination of the small wound in the process of specimen extraction, appropriate surgical techniques, such as averting bile leakage, using a specimen retrieval bag and minimal tumor treatment, are necessary.

In our study, laparoscopic hemihepatectomy with caudate lobectomy was performed in 14 (65.6\%) patients, with the right lobe resected in 6 patients and the left lobe resected in 8 patients. The anatomical characteristics of the hepatic duct at least partly affect the difficulty of hepatectomy. The right hepatic duct is short and has a variable position, which increases the complexity of right hemihepatectomy, whereas the left hepatic duct is more predictable and longer in length. This makes resection of the left half of the liver more straightforward. Among the 24 patients who underwent complete laparoscopic radical resection, 3 patients with a Bismuth Illa cholangiocarcinoma and 2 patients with a Bismuth II cholangiocarcinoma underwent only right hemihepatectomy. However, they all underwent RO resection. In fact, the bile of the caudate lobe gen- erally drains into the biliary confluence or left biliary tree. The hepatic duct of the caudate lobe has a close anatomical relationship with the left hepatic artery. Therefore, en bloc caudate lobectomy should be performed for all left-sided and centrally located tumors but only selectively for right-sided tumors if the duct of the caudate lobe joins the biliary system from the left side. Our experience partly supports this surgical strategy [10]. In the current study, we followed this strategy to develop individualized operation plans. In fact, following these criteria for caudate lobectomy may not only minimize the risk of biliary leak but also reduce the risk of local recurrence from a positive margin, but further clinical research is needed for validation.

\section{Conclusions}

Laparoscopic radical resection for $\mathrm{HC}$ is presently at an early stage but is rapidly developing. From the current study, it is obvious that favorable perioperative outcomes could be achieved by experienced hepatobiliary surgeons, and the benefits of laparoscopic surgery could be provided to highly selected patients. However, laparoscopic radical resection for $\mathrm{HC}$ remains a technical challenge, and it should be performed only in centers with sufficient experience in advanced laparoscopic hepatobiliary surgery. A limitation of this current study is that it is a noncomparative and retrospective case series, which may result in a potential bias. However, the current study has demonstrated a promising future. Randomized trials with long-term follow-up should be performed to address the question of whether laparoscopic procedures can achieve the same results as open surgery. The results of this retrospective case series study could form the foundation for a future randomized trial.

\section{Acknowledgments}

Jingdong Li, Yongfu Xiong contributed equally to this work.

\section{Conflict of interest}

The authors declare no conflict of interest.

\section{References}

1. Ito F, Cho CS, Rikkers LF, Weber SM. Hilar cholangiocarcinoma: current management. Ann Surg 2009; 250: 210-8.

2. Mansour JC, Aloia TA, Crane CH, et al. Hilar cholangiocarcinoma: expert consensus statement. HPB (Oxford) 2015; 17: 691-9. 
3. Fisher AV, Ronnekleiv-Kelly SM. Surgical management of hilar cholangiocarcinoma. Curr Surg Rep 2018; 6.

4. Anderson B, Doyle M. Surgical considerations of hilar cholangiocarcinoma. Surg Oncol Clin N Am 2019; 28: 601-17.

5. Cai WK, Sima H, Chen BD, Yang GS. Risk factors for hilar cholangiocarcinoma: a case-control study in China. World J Gastroenterol 2011; 17: 249-53.

6. Chen W, Zheng R, Baade PD, et al. Cancer statistics in China, 2015. CA Cancer J Clin 2016; 66: 115-32.

7. Siegel RL, Miller KD, Jemal A. Cancer statistics, 2019. CA Cancer J Clin 2019; 69: 7-34.

8. Mizuno T, Ebata T, Nagino M. Advanced hilar cholangiocarcinoma: an aggressive surgical approach for the treatment of ad vanced hilar cholangiocarcinoma: Perioperative management, extended procedures, and multidisciplinary approaches. Surg Oncol 2020: 33: 201-6.

9. Cillo U, Fondevila C, Donadon M, et al. Surgery for cholangiocarcinoma. Liver Int 2019; 39 Suppl 1: 143-55.

10. Lidsky ME, Jarnagin WR. Surgical management of hilar cholangiocarcinoma at Memorial Sloan Kettering Cancer Center. Ann Gastroenterol Surg 2018; 2: 304-12.

11. Burke EC, Jarnagin WR, Hochwald SN, et al. Hilar cholangiocarcinoma: patterns of spread, the importance of hepatic resection for curative operation, and a presurgical clinical staging system. Ann Surg 1998; 228: 385-94.

12. Zheng-Rong L, Hai-Bo Y, Xin C, et al. Resection and drainage of hilar cholangiocarcinoma: an 11-year experience of a single center in mainland China. Am Surg 2011; 77: 627-33.

13. Jarnagin WR, Fong $Y$, Dematteo RP, et al. Staging, resectability, and outcome in 225 patients with hilar cholangiocarcinoma. Ann Surg 2001; 234: 507-19.

14. Parikh A, Abdalla E, Vauthey J. Operative considerations in resection of hilar cholangiocarcinoma. HPB 2005; 7: 254-8.

15. Dinant S, Gerhards M, Busch O, et al. The importance of complete excision of the caudate lobe in resection of hilar cholangiocarcinoma. HPB 2005; 7: 263-7.

16. Antoniou SA, Antoniou GA, Antoniou Al, Granderath FA. Past, present, and future of minimally invasive abdominal surgery. JSLS 2015; 19: e2015.00052.

17. Wu CT, Tsai MH, Lu TP, et al Performances evaluation of algorithms for identifying differentially expressed genes in RNA-sec data. Cancer Res 2015; 75: 4852-2.

18. Fretland A, Dagenborg V, Bjørnelv G, et al. Laparoscopic versus open resection for colorectal liver metastases: the OSLO COMET randomized controlled trial. Ann Surg 2018; 267: 199-207.

19. Petersson J, Koedam T, Bonjer H, et al. Bowel obstruction and ventral hernia after laparoscopic versus open surgery for rectal cancer in a randomized trial (COLOR II). Ann Surg 2019; 269: 53-7.

20. Bonjer H, Deijen C, Abis G, et al. A randomized trial of laparoscopic versus open surgery for rectal cancer. N Engl J Med 2015; 372: 1324-32

21. Kanazawa A, Tsukamoto T, Shimizu S, et al. Laparoscopic hepatectomy for liver cancer. Dig Dis 2015; 33: 691-8.

22. Yoon Y, Han H, Cho J, et al. Is laparoscopy contraindicated for gallbladder cancer? A 10-year prospective cohort study. I Am Coll Surg 2015; 221: 847-53.
23. Palanisamy S, Patel N, Sabnis S, et al. Laparoscopic radical cholecystectomy for suspected early gall bladder carcinoma: thinking beyond convention. Surg Endosc 2016; 30: 2442-8.

24. Sujata J, Rana S, Sabina K, et al. Incidental gall bladder carcinoma in laparoscopic cholecystectomy: a report of 6 cases and a review of the literature. J Clin Diagn Res 2013; 7: 85-8.

25. Berger-Richardson D, Chesney T, Englesakis M, et al. Trends in port-site metastasis after laparoscopic resection of incidental gallbladder cancer: a systematic review. Surgery 2017; 161: 618-27.

26. Dorobisz T, Dorobisz K, Chabowski M, et al. Incidental gallbladder cancer after cholecystectomy: 1990 to 2014. Onco Targets Ther 2016; 9: 4913-6.

27. Paolucci V. Port site recurrences after laparoscopic cholecystectomy. J Hepatobiliary Pancreat Surg 2001; 8: 535-543.

28. Nelson H, Sargent D, Wieand H, et al. A comparison of laparoscopically assisted and open colectomy for colon cancer. N Engl J Med 2004; 350: 2050-9.

29. Abu Hilal M, Badran A, Di Fabio F, Pearce N. Pure laparoscopic en bloc left hemihepatectomy and caudate lobe resection in patients with intrahepatic cholangiocarcinoma. J Laparoendosc Adv Surg Technt A 2011; 21: 845-9.

30. Zhang CW, Liu J, Hong DF, et al. Pure laparoscopic radical resection for type IIla hilar cholangiocarcinoma. Surg Endosc 2018; 32: 1581-2.

31. Xu Y, Wang H, Ji W, et al. Robotic radical resection for hilar cholangiocarcinoma: perioperative and long-term outcomes of an initial series. Surg Endosc 2016; 30: 3060-70.

32. Lee W, Han HS, Yoon YS, et al. Laparoscopic resection of hilar cholangiocarcinoma. Ann Surg Treat Res 2015; 89: 228-32.

33. Machado MA, Makdissi FF, Surjan RC, Mochizuki M. Laparoscopic resection of hilar cholangiocarcinoma. J Laparoendosc Adv Surg Tech A 2012; 22: 954-6.

34. Yu H, Wu SD, Chen DX, Zhu G. Laparoscopic resection of bismuth type I and II hilar cholangiocarcinoma: an audit of 14 cases from two institutions. Dig Surg 2011; 28: 44-9.

35. Zhang Y, Dou C, Wu W, et al. Total laparoscopic versus open radical resection for hilar cholangiocarcinoma. Surg Endosc 2019. doi: 10.1007/s00464-019-07211-0.

36. Feng F, Cao X, Liu X, et al. Laparoscopic resection for Bismuth type III and IV hilar cholangiocarcinoma: how to improve the radicality without direct palpation. J Surg Oncol 2019; 120: 1379-85.

37. Nagino M, Ebata T, Yokoyama $Y$, et al Evolution of surgical treatment for perihilar cholangiocarcinoma: a single-center 34-year review of 574 consecutive resections. Ann Surg 2013; 258: $129-40$.

38. Song SC, Choi DW, Kow AW, et al. Surgical outcomes of 230 resected hilar cholangiocarcinoma in a single centre. ANZ I Surg 2013; 83: 268-74.

39. Agarwal AK, Javed A, Kalayarasan R, Sakhuja P. Minimally invasive versus the conventional open surgical approach of a radical cholecystectomy for gallbladder cancer: a retrospective comparative study. HPB 2015; 17: 536-41.

40. Tran TB, Ethun CG, Pawlik TM, et al. Actual 5-year survivors after surgical resection of hilar cholangiocarcinoma. Ann Surg Oncol 2019; 26: 611-8. 
41. Boggi U, Amorese G, Vistoli F, et al. Laparoscopic pancreaticoduodenectomy: a systematic literature review. Surg Endosc 2015; 29: 9-23.

42. Cheek SM, Sucandy I, Tsung A, et al. Evidence supporting laparoscopic major hepatectomy. J Hepatobiliary Pancreat Sci 2016; 23: 257-9.

43. Gao C. Professor Bing Peng: there is no shortcut to anywhere you want to go. Ann Transl Med 2018; 6: 274.

44. Wang M, Peng B, Liu J, et al. Practice patterns and perioperative outcomes of laparoscopic pancreaticoduodenectomy in China: a retrospective multicenter analysis of 1029 patients. Ann Surg 2019. doi: 10.1097/SLA.0000000000003190.

45. Song KB, Kim SC, Lee W, et al. Laparoscopic pancreaticoduodenectomy for periampullary tumors: lessons learned from 500 consecutive patients in a single center. Surg Endosc 2020; 34: 1343-52.

46. Giulianotti PC, Sbrana F, Bianco FM, Addeo P. Robot-assisted laparoscopic extended right hepatectomy with biliary reconstruction. J Laparoendosc Adv Surg Tech A 2010; 20 159-63.

47. Klatskin G. Adenocarcinoma of the hepatic duct at its bifurcation within the porta hepatis. an unusual tumor with distinctive clinical and pathological features. Am J Med 1965; 38: 241-56.

48. Labib PL, Goodchild G, Pereira SP. Molecular pathogenesis of cholangiocarcinoma. BMC Cancer 2019; 19: 185.

49. Krasinskas AM. Cholangiocarcinoma. Surg Pathol Clin 2018; 11: 403-29.

50. Razumilava N, Gores GJ. Cholangiocarcinoma. Lancet 2014; 383: 2168-79.

51. Cho MS, Kim SH, Park SW, et al. Surgical outcomes and predicting factors of curative resection in patients with hilar cholangiocarcinoma: 10-year single-institution experience. J Gastrointest Surg 2012; 16: 1672-9.

52. Ruys AT, Busch OR, Gouma DJ, van Gulik TM. Staging laparoscopy for hilar cholangiocarcinoma: is it still worthwhile? Indian J Surg Oncol 2012; 3: 147-53.

53. Li H, Wei Y. Laparoscopic extended left hemi-hepatectomy plus caudate lobectomy for caudate lobe hepatocellular carcinoma. J Gastrointest Surg 2019; 23: 617.

54. Inoue $\mathrm{Y}$, Ishii M, Tsuchimoto $\mathrm{Y}$, et al. Comparison of resection site of standardized laparoscopic hepatic tumor resection. Videosurgery Miniinv 2018; 13: 333-41.

55. Ho KM, Han HS, Yoon YS, et al. Laparoscopic total caudate lobectomy for hepatocellular carcinoma. J Laparoendosc Adv Surg Tech A 2017; 27: 1074-8.

56. Oh D, Kwon CH, Na BG, et al. Surgical techniques for totally laparoscopic caudate lobectomy. I Laparoendosc Adv Surg Tech A 2016; 26: 689-92.

57. Franken LC, van der Poel MJ, Latenstein AEJ, et al. Minimally invasive surgery for perihilar cholangiocarcinoma: a systematic review. J Robot Surg 2019; 13: 717-27.

58. Kasai M, Cipriani F, Gayet B, et al. Laparoscopic versus open major hepatectomy: a systematic review and meta-analysis of individual patient data. Surgery 2018; 163: 985-95.
59. Livingston EH, Rege RV. A nationwide study of conversion from laparoscopic to open cholecystectomy. Am J Surg 2004; 188: 205-11.

60. Paolucci V, Schaeff B, Schneider M, Gutt C. Tumor seeding following laparoscopy: international survey. World J Surg 1999; 23: 989-995.

Received: 22.04.2020, accepted: 14.06.2020. 